\title{
Domination in commuting graph and its complement
}

\author{
E. VATANDOOST AND Y. GOLKHANDYPOUR \\ Department of mathematics, International university of imam Khomeini, Qazvin, Iran \\ vatandoost@sci.ikiu.ac.ir and y.golkhandypour@edu.ikiu.ac.ir
}

\begin{abstract}
For each non-commutative ring $R$, the commuting graph of $R$ is a graph with vertex set $R \backslash Z(R)$ and two vertices $x$ and $y$ are adjacent if and only if $x \neq y$ and $x y=y x$. In this paper, we consider the domination and signed domination numbers on commuting graph $\Gamma(R)$ for non-commutative ring $R$ with $Z(R)=\{0\}$. For a finite ring $R$, it is shown that $\gamma(\Gamma(R))+\gamma(\bar{\Gamma}(R))=|R|$ if and only if $R$ is non-commutative ring on 4 elements. Also we determine the domination number of $\Gamma\left(\prod_{i=1}^{t} R_{i}\right)$ and commuting graph of non-commutative ring $R$ of order $p^{3}$, where $p$ is prime. Moreover we present an upper bound for signed domination number of $\Gamma\left(\prod_{i=1}^{t} R_{i}\right)$.

keywords: Noncommutative ring; Commuting graph; Domination number; signed domination number.
\end{abstract}

\section{INTRODUCTION}

L Et $R$ be a non-commutative ring, $Z(R)$ denoted the center of $R$ and for $a \in R$, $C(a)$ denotes the centeralizer of $a$ in $R$. The commuting graph of $R$, denoted by $\Gamma(R)$, is a graph with vertex set $R \backslash Z(R)$ and joined two vertices $x$ and $y$ if and only if $x \neq y$ and $x y=y x$. This graph was introduced by Akbari et al. [2], and the complement of commuting graph of $R$ is denoted by $\bar{\Gamma}(R)$. See [1], [2], [3], [4] and [14] for more details.

Let $G=(V, E)$ be a simple graph comprising a set $V(G)$ of vertices together with a set $E(G)$ of edges. A graph $G$ is said to be connected if each pair of vertices are joined by a walk. The number of edges of the shortest walk joining $v$ and $u$ is called the distance between $v$ and $u$ and denoted by $d(v, u)$. The maximum value of the distance function in a connected graph $G$ is called the diameter of $G$ and denoted by $\operatorname{diam}(G)$. The neigbours of a vertex $v \in V(G)$ is the set of edges incident to the $v$ and denoted by $N(v)$ and $|N(v)|=\operatorname{deg}(v)$. The maximum degree of a graph $G$ denoted by $\Delta(G)$, and the minimum degree of a graph $G$ denoted by $\delta(G)$, are the maximum and minimum degree of its vertices. The complete graph, $K_{n}$, is a graph with $n$ vertices in which each pair of vertices are adjacent. A complete bipartite graph is a graph whose vertices can be divided into two disjoint set $A$ and $B$ such that each edge is incident to a vertex in $A$ and a vertex in $B$ and denoted by $K_{n, m}$ where $|A|=n$ and $|B|=m$. The strong product, $G \otimes H$, of graphs $G$ and $H$ is a graph whose structure is as follows:

i) The vertex set of $G \otimes H$ is the Cartesian product $V(G) \times V(H)$.

ii) Any two distinct vertices $\left(v, v^{\prime}\right)$ and $\left(u, u^{\prime}\right)$ are adjacent in $G \otimes H$ if and only if $v$ is adjacent to $u$ in $G$ and $v^{\prime}=u^{\prime}$, or $v=u$ and $v^{\prime}$ is adjacent to $u^{\prime}$ in $H$, or $v$ is adjacent to $u$ in $G$ and $v^{\prime}$ is adjacent to $u^{\prime}$ in $H$.

The corona $G=G_{1} \circ G_{2}$ is the graph formed from one copy of $G_{1}$ and $\left|V\left(G_{1}\right)\right|$ copies of $G_{2}$ where the $i$ th vertex of $G_{1}$ is adjacent to every vertex in the $i$ th copy of $G_{2}$.

A dominating set of $G$ is a subset $D$ of $V(G)$ such that every vertex not in $D$ is adjacent to at least one vertex in $\mathrm{D}$. The domination number of $G$ is the number of vertices in a minimal dominating set for $G$ and denoted by $\gamma(G)$. See [8], [9] and [15] for more details.

The closed neighbour of $v$, denoted by $N[v]$, is the set $N(v) \cup\{v\}$. A function $f: V \rightarrow\{-1,1\}$ is a signed dominating function if for every vertex $v \in V(G)$, the closed neighbour of $v$ 
contains more vertices with function value 1 than with -1 . Thus $f$ is a signed dominating function if $f[v] \geq 1$ for all $v \in V(G)$, where $f[v]=\sum_{v \in V(G)} f(v)$. The weight of $f$, denoted $f(G)$, is the sum of the function value of all vertices in $G$, i.e., $f(G)=\sum_{x \in V(G)} f(x)$. The signed domination number of $G$, denoted $\gamma_{s}(G)$, is the minimum weight of signed dominating functions of $G$. Also the set of vertices with function value -1 is denoted by $V^{-}(G)$.

In this paper, we consider the domination and signed domination numbers on commuting graph $\Gamma(R)$ for non-commutative ring $R$ with $Z(R)=\{0\}$. For a finite ring $R$, it is shown that $\gamma(\Gamma(R))+\gamma(\bar{\Gamma}(R))=|R|$ if and only if $R$ is the non-commutative ring on 4 elements. Also we determine the domination number of $\Gamma\left(\prod_{i=1}^{t} R_{i}\right)$ and commuting graph of non-commutative ring $R$ of order $p^{3}$, where $p$ is prime. Moreover we present an upper bound for the signed domination number of $\Gamma\left(\prod_{i=1}^{t} R_{i}\right)$. The main results in this paper are the following.

Theorem A. Let $R$ be a non-commutative ring of order $n$ and $Z(R)=\{0\}$. Then

i) $\gamma(\Gamma(R))+\gamma(\bar{\Gamma}(R))=n$ if and only if $R$ is isomorphic with one of the following rings:

$E=\left\langle x, y: 2 x=2 y=0, x^{2}=x, y^{2}=\right.$ $y, x y=x, y x=y\rangle$

$F=\left\langle x, y: 2 x=2 y=0, x^{2}=x, y^{2}=\right.$ $y, x y=y, y x=x\rangle$.

ii) $\gamma(\Gamma(R))+\gamma(\bar{\Gamma}(R)) \neq n-1$.

iii) $\gamma(\Gamma(R))+\gamma(\bar{\Gamma}(R))=n-2$ if and only if $n$ is even and $\Gamma(R)=K_{3} \cup(n-4) K_{1}$.

Theorem B. Let $\mathrm{R}$ be a non-commutative ring of order $p^{3}$ and $Z(R)=\{0\}$. Then

i) $\gamma(\Gamma(R))=p^{2}+p+1$.

or

ii) $\gamma(\Gamma(R))=\ell_{1}+\ell_{2}$, where $\ell_{1}$ and $\ell_{2}$ satisfy in $\ell_{1}+(p+1) \ell_{2}=p^{2}+p+1$.

Theorem C. Let $R_{i},(1 \leq i \leq t)$, be noncommutative ring of order $n_{i}$ and $Z\left(R_{i}\right)=\{0\}$. Then $\gamma\left(\Gamma\left(\prod_{i=1}^{t} R_{i}\right)\right)=\operatorname{Min}_{1 \leq i \leq t}\left(\gamma\left(\Gamma\left(R_{i}\right)\right)\right)$.

Theorem D. Let $R$ be a non-commutative ring of order $n$ and $Z(R)=\{0\}$.

i) Let $\mathrm{n}$ be even. Then $\gamma_{s}(\Gamma(R))=n-1$ if and only if $R$ is isomorphic with one of the following rings:

$E=\langle x, y| 2 x=2 y=0, x^{2}=x, y^{2}=y, x y=$ $x, y x=y\rangle$

$F=\langle x, y| 2 x=2 y=0, x^{2}=x, y^{2}=y, x y=$ $y, y x=x\rangle$.

ii) Let $\mathrm{n}$ be odd. Then $\gamma_{s}(\Gamma(R))=n-1$ if and only if $\Gamma(R)$ is the union of $\frac{n-1}{2}$ copies of $P_{2}$.

Theorem E. Let $R_{i}, 1 \leq i \leq t$ be noncommutative ring such that $\left|R_{i}\right|=n_{i}$ and $Z\left(R_{i}\right)=\{0\}$. Also, let $\delta_{i}$ be minimum degree of $\Gamma\left(R_{i}\right)$.

i) If for all $1 \leq i \leq t, \delta_{i}$ is odd, then $\gamma_{s}\left(\Gamma\left(\prod_{i=1}^{t} R_{i}\right)\right) \leq \prod_{i=1}^{t} n_{i}-\prod_{i=1}^{t}\left(\delta_{i}+2\right)+2$.

ii) Otherwise, $\gamma_{s}\left(\Gamma\left(\prod_{i=1}^{t} R_{i}\right)\right) \leq \prod_{i=1}^{t} n_{i}$ $\prod_{i=1}^{t}\left(\delta_{i}+2\right)+1$.

\section{Preliminaries}

First we give some facts that are needed in the section III.

\subsection{On commuting graph}

Lemma 2.1.1 Let $R$ be a non-commutative ring of order $n$ and $Z(R)=\{0\}$. If $\bar{\Gamma}(R)$ contains $a$ vertex of degree $k$, then $k>\frac{n-1}{2}$.

Proof. On the contrary, let $v$ be a vertex of degree $k$ in $\bar{\Gamma}(R)$ such that $k \leq \frac{n-1}{2}$. So $|C(v)| \geq \frac{n-1}{2}+1$. Thus $|C(v)| \nmid n$, which is impossible. Hence $\operatorname{deg}(v)>\frac{n-1}{2}$, for each $v \in \bar{\Gamma}(R)$.

Lemma 2.1.2 Let $R$ be a non-commutative ring of order $n$ and $Z(R)=\{0\}$. Then $\Gamma(R)$ is not a cycle. Also, $\Gamma(R)$ does not have $C_{4}$ as a component.

Proof. On the contrary, let $\Gamma(R)=C_{n-1}$ and $v_{1}, \ldots, v_{n-1} \in V\left(C_{n-1}\right)$, such that $v_{i} \in$ $N\left(v_{i+1}\right)$. So $C\left(v_{i}\right)=\left\{0, v_{i-1}, v_{i}, v_{i+1}\right\}$. Since $C\left(v_{i}\right)$ is a subgroup of $(R,+), v_{i-1}+v_{i+1} \in$ $C\left(v_{i}\right)$. If $v_{i-1}+v_{i+1}=0$, then $v_{i-1}=-v_{i+1}$. It follows that $v_{i-2} \in N\left(v_{i+1}\right)$, which is impossible. If $v_{i-1}+v_{i+1} \in\left\{v_{i-1}, v_{i+1}\right\}$, then $v_{i-1}=0$ or $v_{i+1}=0$, a contradiction. Thus $v_{i-1}+$ 
$v_{i+1}=v_{i}$. Also, $C\left(v_{i-1}\right)=\left\{0, v_{i-1}, v_{i-2}, v_{i}\right\}$. Similarly, $v_{i-2}+v_{i-1}=v_{i}$. Hence $v_{i-2}=v_{i+1}$, which is a contradiction. Therefore $\Gamma(R)$ is not a cycle.

Also let $\Gamma(R)$ has $C_{4}$ as a component and let $V\left(C_{4}\right)=\left\{x_{1}, x_{2}, x_{3}, x_{4}\right\}$ such that $x_{1} \notin N\left(x_{2}\right)$. Then $\left|C\left(x_{1}\right) \cap C\left(x_{2}\right)\right|=3$, which is impossible.

Lemma 2.1.3 Let $R$ be a non-commutative ring of order $n$ and $Z(R)=\{0\}$. Then $\Gamma(R)$ does not have both an isolated vertex and a vertex of degree one.

Proof. On the contrary, let $x$ be an isolated vertex and $y$ be a vertex of degree 1 in $\Gamma(R)$. So $C(x)=\{0, x\}$ and $C(y)=\{0, y,-y\}$. If $C(x)+C(y)=H$, then $H$ is a subgroup of $(R,+)$ and $|H|=6$. Since $x+y \in H$ and $O(x+y)=6,(H,+)$ is a cyclic group. Thus $C(x+y)=H$. It follows that $K_{5}$ is a subgraph of commuting graph. So $x$ is not an isolated vertex, which is contradiction.

Theorem 2.1.4 [14] Let $R$ be a non-commutative finite ring with $|R|>4$. Then $\operatorname{diam}(\bar{\Gamma}(R))=2$.

Theorem 2.1.5 [14] Let $R$ be a non-commutative ring. Then $\bar{\Gamma}(R)$ is not a complete bipartite graph.

Lemma 2.1.6 Let $R$ be a non-commutative ring of order $n$ and $Z(R)=\{0\}$. Then $n \neq 6$.

Proof. On the contrary, let $n=6$. By Lemma 2.1.1. $\delta(\bar{\Gamma}(R)) \geq 3$. If $\delta(\bar{\Gamma}(R))=4$, then $\bar{\Gamma}(R)=K_{5}$, contrary to Theorem 2.1.4 So $\delta(\bar{\Gamma}(R))=3$. Since there is no 3-regular graph on 5 vertices, $\Delta(\bar{\Gamma}(R))=4$. Hence $\bar{\Gamma}(R)$ is one of the graphs that are shown in figure 1 .

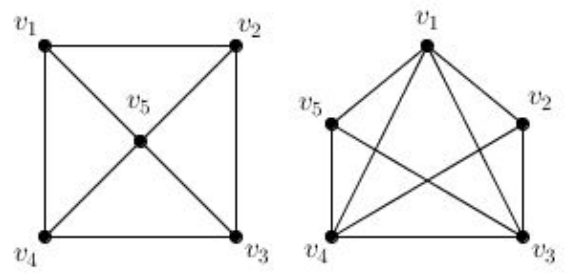

fig1.
In both graphs, $\Gamma(R)$ is a union of isolated vertices and copies of $P_{2}$, contrary to Lemma 2.1.3. Therefore $n \neq 6$.

Corollary 2.1.7 Let $R$ be a non-commutative ring of order $n$ and $Z(R)=\{0\}$. Then $n=4$ or $n \geq 8$.

Lemma 2.1.8 Let $R$ be a ring of order $p$, where $p$ is prime. Then $R$ is a commutative ring.

Proof. The proof is straightforward.

Theorem 2.1.9 [14] Let $R$ be a non-commutative ring of order $p^{2}$, where $p$ is a prime number. Then $R$ is one of the following rings.

$E=\left\langle x, y: p x=p y=0, x^{2}=x, y^{2}=\right.$ $y, x y=x, y x=y\rangle$

$F=\left\langle x, y: p x=p y=0, x^{2}=x, y^{2}=\right.$ $y, x y=y, y x=x\rangle$.

Theorem 2.1.10 [14] Let $R$ be a non-commutative finite ring with $\operatorname{diam}(\bar{\Gamma}(R))=1$. Then $R$ is of type E or F (see Theorem 2.1.9).

Theorem 2.1.11 [2] For any non-commutative ring $R$ and $x, y \in V(\bar{\Gamma}(R))$, there is a path between $x$ and $y$ in $\bar{\Gamma}(R)$ whose length is at most two.

Lemma 2.1.12 Let $R$ be a finite ring of order $p^{2}$ and $Z(R) \neq\{0\}$. Then $R$ is commutative.

Proof. On the contrary, let $R$ be a noncommutative ring. It follows immediately that $|Z(R)|=p$. Clearly, for any $x \in R \backslash Z(R)$, $|C(x)|>p$ and $|C(x)| \mid p^{2}$. So $|C(x)|=p^{2}$ and $x \in Z(R)$, a contradiction. Therefore $R$ is commutative ring.

Lemma 2.1.13 Let $R$ be a non-commutative ring and $x, y \in V(\Gamma(R))$ such that $C(x)$ and $C(y)$ are commutative. If $y \in C(x)$, then $C(x)=C(y)$.

Proof. The proof is straightforward.

Theorem 2.1.14 [17] (Scorza) Let $\left\{A_{i}: 1 \leq\right.$ $i \leq 3\}$ be an irredundant cover with core-free intersection $D$ for a group $G$. Then $D=1$ and $G \cong Z_{2} \oplus Z_{2}$. 


\subsection{On domination number}

Lemma 2.2.1 [12] Let $G$ be a graph on $n$ vertices. Then $\gamma(G)=1$ if and only if $\Delta(G)=n-1$.

Theorem 2.2.2 [13] Let $G$ be a graph on $n$ vertices. Then

i) $\gamma(G)+\gamma(\bar{G}) \leq n+1$.

ii) $\gamma(G) \gamma(\bar{G}) \leq n$.

Theorem 2.2.3 [7] Let $G$ be a graph without isolated vertices on $n$ vertices such that $n$ is even. Then $\gamma(G)=\frac{n}{2}$ if and only if the components of $G$ are $\mathrm{C}_{4}$ or $\mathrm{H} \circ \mathrm{K}_{1}$ where $\mathrm{H}$ is a connected graph.

Theorem 2.2.4 [15] Let $G$ be a graph with no isolated vertex. Then $\gamma(G) \leq \frac{n}{2}$.

Theorem 2.2.5 [5], [18] For any graph $G$,

$$
\left\lceil\frac{n}{1+\Delta(G)}\right\rceil \leq \gamma(G) \leq n-\Delta(G) .
$$

Theorem 2.2.6 [16] If a graph $G$ has no isolated vertices, then

$$
\gamma(G) \leq \frac{n+2-\delta(G)}{2} .
$$

\subsection{On signed domination number}

Lemma 2.3.1 [10] A graph $G$ has $\gamma_{s}(G)=n$ if and only if every $v \in G$ is either isolated, an endvertex or adjacent to an endvertex.

Lemma 2.3.2 Let $G$ be a graph on $n$ vertices and $\alpha$ be an odd number. Then $\gamma_{s}(G) \neq n-\alpha$.

Proof. The proof is straightforward.

Theorem 2.3.3 [11] Let $K_{n}$ be a complete graph on $n$ vertices. Then

$$
\gamma_{s}\left(K_{n}\right)= \begin{cases}2 & n \text { be even } \\ 1 & n \text { be odd }\end{cases}
$$

Lemma 2.3.4 Let $G$ be a graph with $\delta(G) \geq 6$. Then $\left|V^{-}(G)\right| \geq 3$.

Proof. Let $v \in V(G)$ and $\operatorname{deg}(v)=\delta(G) \geq 6$. If $N(v)=\left\{v_{1}, \ldots, v_{\delta(G)}\right\}$, then consider the function $f: V(G) \rightarrow\{-1,1\}$ for which $f(v)=$ $f\left(v_{1}\right)=f\left(v_{2}\right)=-1$. Clearly, $f[w] \geq 1$ where $w \in\left\{v, v_{1}, v_{2}\right\}$. So $\left|V^{-}(G)\right| \geq 3$.
Lemma 2.3.5 Let $R$ be a non-commutative ring of order 8 and $Z(R)=\{0\}$. Then $\gamma_{s}(\bar{\Gamma}(R))=1$.

Proof. Let $v \in V(\bar{\Gamma}(R))$ such that $\operatorname{deg}(v)=k$. By Lemma 2.1.1. $k \geq 4$. If $k=5$, then $|C(v)| \nmid|R|$, which is a contradiction. Hence $k \in\{4,6\}$. If $\delta(\bar{\Gamma}(R))=6$, then $\bar{\Gamma}(R)=K_{7}$, contrary to Theorem 2.1.4.

Let $\delta(\bar{\Gamma}(R))=4$. Then $\Delta(\bar{\Gamma}(R)) \in\{4,6\}$. If $\Delta(\bar{\Gamma}(R))=4$, then $\bar{\Gamma}(R)$ is a 4-regular graph on 7 vertices, which are depicted in figure 2 .

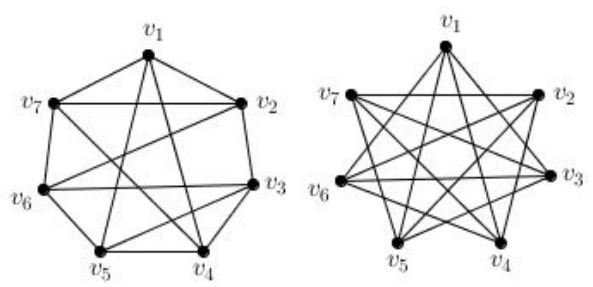

fig 2 .

In both graphs, $\Gamma(R)=C_{7}$, contrary to Lemma 2.1 .2

Let $\Delta(\bar{\Gamma}(R))=6$. Then $\bar{\Gamma}(R)$ has at least two vertices $v$ and $u$ of degree 4 . We consider the following two cases.

Case 1. Let $v \in N(u)$ in $\bar{\Gamma}(R)$. Then $C(v)=$ $\left\{0, v, x_{1}, x_{2}\right\}$ and $C(u)=\left\{0, u, y_{1}, y_{2}\right\}$. So $|C(v) \cap C(u)| \in\{1,2\}$.

If $|C(v) \cap C(u)|=1$, then $|C(v)+C(u)|>|R|$, which is impossible.

Let $|C(v) \cap C(u)|=2, C(v)=\left\{0, v, x_{1}, x_{2}\right\}$ and $C(u)=\left\{0, u, x_{1}, y_{2}\right\}$. Thus $x_{1} \notin N(v) \cup N(u)$, $y_{2} \in N(v)$ and $x_{2} \in N(u)$ in $\bar{\Gamma}(R)$ and there exist $z_{1}, z_{2} \in V(\bar{\Gamma}(R))$ such that $z_{1}, z_{2} \in N(v) \cap$ $N(u)$. This case will be divided into 2 subcases. Subcase i. If $\operatorname{deg}\left(x_{1}\right)=6$, then $x_{1} \in N(v) \cap$ $N(u)$, a contradiction.

Subcase ii. Let $\operatorname{deg}\left(x_{1}\right)=4$. Since $x_{1} \notin N(v) \cup$ $N(u)$, it follows that $\left\{y_{2}, x_{2}, z_{1}, z_{2}\right\} \subseteq N\left(x_{1}\right)$. Similarly, since $y_{2} \in N(v)$ and $x_{2} \in N(u)$, it follows that $\left\{z_{1}, z_{2}\right\} \subseteq N\left(y_{2}\right) \cap N\left(x_{2}\right)$. Thus $\operatorname{deg}\left(x_{2}\right)=\operatorname{deg}\left(y_{2}\right)=4$. We claim that $z_{1} \notin$ $N\left(z_{2}\right)$ in $\bar{\Gamma}(R)$. For let $z_{1} \in N\left(z_{2}\right)$. Then by the above argument, $z_{1}$ and $z_{2}$ have exactly two common neigbours. But $v, u, y_{2}, x_{2} \in$ $N\left(z_{1}\right) \cap N\left(z_{2}\right)$, which is a contradiction. Hence 
$z_{1} \notin N\left(z_{2}\right)$, as claimed. Thus $\operatorname{deg}\left(z_{1}\right)=$ $\operatorname{deg}\left(z_{2}\right)=5$, which is impossible. Therefore this case will not happen.

Case 2. Let $v \notin N(u)$ in $\bar{\Gamma}(R)$. Then $C(v)=$ $C(u)=\{0, v, u, \alpha\}$. So there exist $z_{1}, z_{2}, z_{3}, z_{4} \in$ $V(\bar{\Gamma}(R))$ such that $z_{1}, z_{2}, z_{3}, z_{4} \in N(v) \cap N(u)$ in $\bar{\Gamma}(R)$.

If $\operatorname{deg}(\alpha)=6$ in $\bar{\Gamma}(R)$, then $\alpha \in N(v)$, a contradiction. So $\operatorname{deg}(\alpha)=4$ and $z_{1}, z_{2}, z_{3}, z_{4} \in N(\alpha)$. We claim that $\operatorname{deg}\left(z_{i}\right)=6$, where $1 \leq i \leq 4$. On the contrary, let there exist $1 \leq i \leq 4$ such that $\operatorname{deg}\left(z_{i}\right)=4$. Since $z_{i} \in N(v)$, as in the Case 1 , $z_{i}$ and $v$ should have two common neigbours. Hence $\operatorname{deg}\left(z_{i}\right)=5$, which is a contradiction. Thus $\operatorname{deg}\left(z_{i}\right)=6$.

Consider the function $f: V(\bar{\Gamma}(R)) \rightarrow\{-1,1\}$ for which $f(v)=f(u)=f(\alpha)=-1$ and $f\left(z_{i}\right)=1$, where $1 \leq i \leq 4$. Clearly, $f[w] \geq$ 1 , for all $w \in V(\bar{\Gamma}(R))$. This implies that $\left|V^{-}(\bar{\Gamma}(R))\right| \geq 3$.

If $g$ is a signed domination function on $\bar{\Gamma}(R)$ and $|\{w ; g(w)=-1\}|>3$, then $g[\ell]=-1$ for some $\ell \in\left\{z_{1}, z_{2}, z_{3}, z_{4}\right\}$, which is a contradiction. Therefore $\left|V^{-}(\bar{\Gamma}(R))\right|=3$, and this completed the proof.

Lemma 2.3.6 Let $R$ be a non-commutative ring of order $2 p$, where $p$ is an odd prime and $Z(R)=\{0\}$. Then $\gamma_{s}(\bar{\Gamma}(R))=2$.

proof. Let $|R|=2 p$. Then by Lemma 2.1.6 $p>$ 3. Let $v \in V(\bar{\Gamma}(R))$ such that $\operatorname{deg}(v)=k$. By Lemma 2.1.1. $k \geq p$. Clearly, if $k \notin\{p, 2 p-2\}$, then $|C(v)| \nmid 2 p$, which is impossible. Thus $k \in\{p, 2 p-2\}$. If $\delta(\bar{\Gamma}(R))=2 p-2$, then $\bar{\Gamma}(R)$ is a complete graph, contrary to Theorem 2.1.4 If $\Delta(\bar{\Gamma}(R))=p$, then we have a $p$-regular graph on $2 p-1$ vertices, which is impossible. So $\Delta(\bar{\Gamma}(R))=2 p-2$ and $\bar{\Gamma}(R)$ has at least two vertices $v$ and $u$ of degree $p$. The following two cases will be considered.

Case 1. Let $v \in N(u)$ in $\bar{\Gamma}(R)$. Then $C(v)=\left\{0, v, x_{1}, \ldots, x_{p-2}\right\}$ and $C(u)=$ $\left\{0, u, y_{1}, \ldots, y_{p-2}\right\}$. Thus $|C(v) \cap C(u)|=1$ and so $|C(v)+C(u)|=p^{2}>2 p$, which is impossible.

Therefore this case will not happen.

Case 2. Let $v \notin N(u)$ in $\bar{\Gamma}(R)$. Then $C(v)=\left\{0, v, u, x_{1}, \ldots, x_{p-3}\right\}$ and $C(u)=$ $\left\{0, u, v, y_{1}, \ldots, y_{p-3}\right\}$. Thus $|C(v) \cap C(u)|=p$ and so $C(v)=C(u)=\left\{0, v, u, z_{1}, z_{2}, \ldots, z_{p-3}\right\}$. Hence there exist $\alpha_{1}, \ldots, \alpha_{p} \in V(\bar{\Gamma}(R))$ such that $\alpha_{1}, \ldots, \alpha_{p} \in N(v) \cap N(u)$ in $\bar{\Gamma}(R)$. Obviously, $z_{1}, z_{2}, \ldots, z_{p-3} \notin N(v) \cup N(u)$. We claim that induced subgraph on $\left\{z_{1}, z_{2}, \ldots, z_{p-3}\right\}$ is empty. On the contrary, let $z_{1} \in N\left(z_{2}\right)$. By Case 1, $\operatorname{deg}\left(z_{1}\right) \neq p, \operatorname{deg}\left(z_{2}\right) \neq p$ or $\operatorname{deg}\left(z_{1}\right), \operatorname{deg}\left(z_{2}\right) \neq p$. Whithout loss of generality, let $\operatorname{deg}\left(z_{1}\right)=2 p-2$. Since $\left|\left\{z_{2}, z_{3}, \ldots, z_{p-3}\right\}\right|+\left|\left\{\alpha_{1}, \ldots, \alpha_{p}\right\}\right|=2 p-4$, it follows that $\operatorname{deg}\left(z_{1}\right) \leq 2 p-4$, which is a contradiction. Thus $z_{1} \notin N\left(z_{2}\right)$. Similarly, $z_{i} \notin N\left(z_{j}\right)$, where $1 \leq i, j \leq p-3$, and so induced subgraph on $\left\{z_{1}, z_{2}, \ldots, z_{p-3}\right\}$ is empty, as claimed. Hence $\operatorname{deg}\left(z_{i}\right)=p$, where $1 \leq i \leq$ $p-3$ and by Case 1 , for each $\ell \in\left\{\alpha_{1}, \ldots, \alpha_{p}\right\}$, $\operatorname{deg}(\ell)=2 p-2$.

Consider the function $f: V(\bar{\Gamma}(R)) \rightarrow\{-1,1\}$ for which $f(v)=f(u)=f\left(z_{i}\right)=-1$, where $1 \leq i \leq p-3$ and $f\left(\alpha_{j}\right)=1$, where $1 \leq j \leq p$. Clearly, $f[w] \geq 1$, for all $w \in V(\bar{\Gamma}(R))$. This implies that $\left|V^{-}(\bar{\Gamma}(R))\right| \geq p-1$.

If $g$ is a signed domination function on $\bar{\Gamma}(R)$ and $|\{w ; g(w)=-1\}|>p-1$, then $g[\ell]=-1$ for some $\ell \in\left\{\alpha_{1}, \ldots, \alpha_{p}\right\}$, which is contradiction. Therefore $\left|V^{-}(\bar{\Gamma}(R))\right|=p-1$ and this completed the proof.

\section{Main Results}

In this section we prove our main results.

\subsection{Domination number in $\Gamma(R)$}

Theorem 3.1.1 Let $R$ be a non-commutative ring of order odd number $n$ and $Z(R)=\{0\}$. Then $\gamma(\Gamma(R))=\frac{n-1}{2}$ if and only if $\Gamma(R)$ is a union of $\frac{n-1}{2}$ copies of $P_{2}$. Also $n=3^{k}$ for $k>1$.

Proof. Let $\gamma(\Gamma(R))=\frac{n-1}{2}$. Since $n$ is odd, $\Gamma(R)$ does not have isolated vertex. By Theorem 2.2.3. the components of $\Gamma(R)$ are $C_{4}$ or $H \circ K_{1}$ where $H$ is a connected graph. By Lemma 2.1.2. $\Gamma(R)$ does not have $C_{4}$ as a component. Hence the components of $\Gamma(R)$ are $H \circ K_{1}$. Let $x$ be an endvertex in $\Gamma(R)$ and $x \in N(y)$. Then $C(x)=\{0, x, y\}$. If $O(x)=2$, then $n$ is even, 
which is false. So $O(x) \neq 2$. If $-x \neq y$, then $x$ is adjacent to $-x$ in $\Gamma(R)$, which is a contradiction. Hence $-x=y$ and so $\operatorname{deg}(y)=1$. Thus $\Gamma(R)$ is union of $\frac{n-1}{2}$ copies of $P_{2}$. Therefore for every $0 \neq z \in R, C(z)=\{0, z,-z\}$ and so $O(z)=3$. Hence $n=3^{k}$ for $k>1$. The proof of converse is easy.

Corollary 3.1.2 Let $R$ be a non-commutative ring of order $n$ such that $n$ and $|Z(R)|=t$ are odd. Then $\gamma(\Gamma(R))=\frac{n-t}{2}$ if and only if $\Gamma(R)$ is the union of $\frac{n-t}{2}$ copies of $P_{2}$.

Lemma 3.1.3 Let $R$ be a non-commutative ring of order $n$ and $Z(R)=\{0\}$. Then $\gamma(\Gamma(R)) \geq 3$.

Proof. On the contrary, let $\gamma(\Gamma(R))<3$. If $\gamma(\Gamma(R))=1$, then there exist $x \in R \backslash Z(R)$ such that $C(x)=R$, which is impossible. Let $\gamma(\Gamma(R))=2$ and $D=\{x, y\}$ be a dominating set in $\Gamma(R)$. Then $R=C(x) \cup C(y)$. Hence $C(x) \subseteq C(y)$ or $C(y) \subseteq C(x)$. Without loss of generality, let $C(x) \subseteq C(y)$. Then $R=C(y)$ and so $y \in Z(R)$, which is a contradiction.

Lemma 3.1.4 Let $R$ be a non-commutative ring of order $n$ and $Z(R)=\{0\}$. Then $\gamma(\Gamma(R))=3$ if and only if $R$ is isomorphic with one of the following rings:

$E=\left\langle x, y: 2 x=2 y=0, x^{2}=x, y^{2}=y, x y=\right.$ $x, y x=y\rangle$

$F=\left\langle x, y: 2 x=2 y=0, x^{2}=x, y^{2}=y, x y=\right.$ $y, y x=x\rangle$.

Proof. It is not hard to see that $\gamma(\Gamma(E))=$ $\gamma(\Gamma(F))=3$. Conversely, let $\gamma(\Gamma(R))=3$ and $D=\{x, y, z\}$ be a dominating set in $\Gamma(R)$. Then $R=C(x) \cup C(y) \cup C(z)$. By Theorem 2.1.14. $(R,+) \cong Z_{2} \oplus Z_{2}$. On the other hand, exactly $E$ and $F$ are non-commutative rings between all rings of order four.

Corollary 3.1.5 Let $R$ be a non-commutative ring of order $n$ and $Z(R) \neq\{0\}$. Then $\gamma(\Gamma(R)) \geq 4$.

Lemma 3.1.6 Let $R$ be a non-commutative ring of order $n$ with $Z(R)=\{0\}$. If $\gamma(\bar{\Gamma}(R))=1$, then $n=2^{t}$ for positive integer $t$.
Proof. Let $D=\{x\}$ be a dominating set in $\bar{\Gamma}(R)$. So $x$ is an isolated vertex in $\Gamma(R)$, and so $O(x)=2$. Hence $n=2 k$. On the contrary, let $p \mid n$, where $p$ is an odd prime. So there exist $y \in R$ such that $O(y)=p$. Hence $p y=0, p x=x$ and $2 y \neq 0$. So $2 y p x=0$. Thus $2 y \in C(x)$ and so $2 y=x$. It follows that $O(y)=4$, which is a contradiction. Therefore $n=2^{t}$.

Lemma 3.1.7 Let $R$ be a non-commutative ring of order odd number $n, Z(R)=\{0\}$ and $3 \nmid n$. Then $3<\gamma(\Gamma(R))<\frac{n-1}{2}$.

Proof. Since $n$ is odd, $\Gamma(R)$ does not have isolated vertex. So by Theorem 2.2.4. $\gamma(\Gamma(R)) \leq$ $\frac{n-1}{2}$. If $\gamma(\Gamma(R))=\frac{n-1}{2}$, then by Theorem 3.1.1. $n=3^{k}$ for $k>1$, which is a contradiction. By Lemma 3.1.3, $\gamma(\Gamma(R)) \geq 3$. if $\gamma(\Gamma(R))=3$, then by Lemma 3.1.4. $n=4$, which is impossible. Therefore $3<\gamma(\Gamma(R))<\frac{n-1}{2}$.

Lemma 3.1.8 Let $R$ be a non-commutative ring of order $n$ and $Z(R)=\{0\}$. Then $\gamma(\bar{\Gamma}(R))<\frac{n-1}{2}$.

Proof. Since $\bar{\Gamma}(R)$ is a connected graph, $\gamma(\bar{\Gamma}(R)) \leq \frac{n-1}{2}$. If $\gamma(\bar{\Gamma}(R))=\frac{n-1}{2}$, then by Theorem 2.2.3. $\bar{\Gamma}(R)=C_{4}$ or $\bar{\Gamma}(R)=H \circ K_{1}$ where $H$ is a connected graph. In both cases $\gamma(\Gamma(R))=2$, contrary to Lemma 3.1.3 Therefore $\gamma(\bar{\Gamma}(R))<\frac{n-1}{2}$.

Theorem 3.1.9 Let $R$ be a non-commutative ring and $Z(R) \neq\{0\}$. Then $\gamma(\bar{\Gamma}(R)) \geq 2$.

Proof. On the contrary, let $\gamma(\bar{\Gamma}(R))=1$ and $D=\{x\}$ be a dominating set in $\bar{\Gamma}(R)$. So $C(x)=Z(R) \cup\{x\}$. Let $0 \neq y \in Z(R)$. So $x(y+x)=(y+x) x$, and so $y+x \in C(x)$. If $y+x=x$, then $y=0$, which is impossible. Also if $y+x \in Z(R)$, then $x \in Z(R)$, which is a contradiction. Therefore $\gamma(\bar{\Gamma}(R)) \geq 2$.

Corollary 3.1.10 If $R$ is a non-commutative ring with unity, then $\Gamma(R)$ has no isolated vertices.

\section{Proof of Theorem A.}

i) Let $\gamma(\Gamma(R))+\gamma(\bar{\Gamma}(R))=n$. If $\Gamma(R)$ has no isolated vertex, then $\gamma(\Gamma(R)) \leq \frac{n-1}{2}$, by Theorem 2.2.4 Since $\bar{\Gamma}(R)$ is a connected graph, $\gamma(\bar{\Gamma}(R)) \leq \frac{n-1}{2}$. Thus $\gamma(\Gamma(R))+$ 
$\gamma(\bar{\Gamma}(R)) \leq n-1$, which contradicts the assumption. Hence $\Gamma(R)$ has at least one isolated vertex. Thus $\gamma(\bar{\Gamma}(R))=1$ and so $\gamma(\Gamma(R))=n-1$. Therefore $\Gamma(R)=(n-1) K_{1}$ and $\bar{\Gamma}(R)=K_{n-1}$. By Theorem 2.1.10, $R$ is of type $E$ or $F$. The proof of converse is clear. ii) Let $\gamma(\Gamma(R))+\gamma(\bar{\Gamma}(R))=n-1$. Since $\bar{\Gamma}(R)$ is a connected graph, $\gamma(\bar{\Gamma}(R)) \leq \frac{n-1}{2}$. If $\Gamma(R)$ has no isolated vertex, then by Theorem 2.2.4. $\gamma(\Gamma(R)) \leq \frac{n-1}{2}$. So $\gamma(\Gamma(R))=\gamma(\bar{\Gamma}(R))=\frac{n-1}{2}$, contrary to Lemma 3.1.8. Hence $\Gamma(R)$ has at least one isolated vertex and so $\gamma(\bar{\Gamma}(R))=1$. Thus $\gamma(\Gamma(R))=n-2$. It is easy to see that $\Gamma(R)=(n-3) K_{1} \cup P_{2}$, contrary to Lemma 2.1.3. Therefore $\gamma(\Gamma(R))+\gamma(\bar{\Gamma}(R)) \neq n-1$. iii) Let $\gamma(\Gamma(R))+\gamma(\bar{\Gamma}(R))=n-2$. If $\Gamma(R)$ has no isolated vertex, then by Theorem 2.2.4 $\gamma(\Gamma(R)) \leq \frac{n-1}{2}$. Since $\bar{\Gamma}(R)$ is connected graph, $\gamma(\bar{\Gamma}(R)) \leq \frac{n-1}{2}$. By Lemma 3.1.8. $\gamma(\bar{\Gamma}(R))<\frac{n-1}{2}$. If $\gamma(\bar{\Gamma}(R))=\frac{n-1}{2}-1$, then $\gamma(\Gamma(R))=\frac{n-1}{2}$. By Theorem 3.1.1. $\Gamma(R)$ is a union of $\frac{n-1}{2}$ copies of $P_{2}$. It follows that $\bar{\Gamma}(R)$ is a regular multipartite graph of size two. Hence $\gamma(\bar{\Gamma}(R))=2$, which contradicts the fact that $n$ is not prime. Also if $\gamma(\bar{\Gamma}(R)) \leq \frac{n-5}{2}$, then $\gamma(\Gamma(R))>\frac{n-1}{2}$, contrary to Theorem 2.2 .4

Thus $\Gamma(R)$ has at least one isolated vertex and so $\gamma(\Gamma(R))=n-3$. Let $D=\left\{x_{1}, x_{2}, \ldots, x_{n-3}\right\}$ be a dominating set in $\Gamma(R)$. Then there exist unique vertices $x_{i}, x_{j} \in D$ such that $x_{n-1} \in$ $N\left(x_{i}\right)$ and $x_{n-2} \in N\left(x_{j}\right)$ in $\Gamma(R)$. It is clear that induced subgraph on $D \backslash\left\{x_{i}, x_{j}\right\}$ is empty. Let $A=\left\{x_{i}, x_{j}, x_{n-1}, x_{n-2}\right\}$. The proof will be divided into 2 cases.

Case 1. If $x_{i} \neq x_{j}$, then by Lemma 2.1.3, induced subgraph on $A$ is $C_{4}$, contrary to Lemma 2.1.2.

Case 2. If $x_{i}=x_{j}$, then by Lemma 2.1.3, induced subgraph on $A$ is $K_{3}$ and so $\Gamma(R)=$ $K_{3} \cup(n-4) K_{1}$. Also we has $C\left(x_{n-1}\right)=$ $\left\{0, x_{n-1}, x_{n-2}, x_{i}=x_{j}\right\}$. So $4 \mid n$. Hence $|R|=n$ is even. The proof of converse is clear.

Theorem 3.1.11 Let $R$ be a non-commutative ring of order $p^{2}$ and $Z(R)=\{0\}$. Then $\gamma(\Gamma(R))=$ $p+1$.
Proof. We refer the reader to [[14], Th. 4].

Lemma 3.1.12 Let $R$ be a non-commutative ring of order $p^{3}$ and $Z(R)=\{0\}$. If $x, y \in V(\Gamma(R))$ and $x y \neq y x$, then $C(x) \cap C(y)=\{0\}$.

Proof. Let $z \in V(\Gamma(R))$. Since $C(z)$ is the addition subgroup of $R,|C(z)| \in\left\{p, p^{2}\right\}$. The following 3 cases will be considered.

Case1. Let $|C(x)|=|C(y)|=p$. Then $|C(x) \cap C(y)| \in\{1, p\}$.

If $|C(x) \cap C(y)|=1$, then $C(x) \cap C(y)=\{0\}$. If $|C(x) \cap C(y)|=p$, then $|C(x) \cap C(y)|=$ $|C(x)|$. Since $C(x) \cap C(y) \subseteq C(x), C(x) \cap$ $C(y)=C(x)$. Thus $C(x) \subseteq C(y)$. This contradicts the fact that $y \notin C(x)$.

Case 2. Let $|C(x)|=|C(y)|=p^{2}$. Then $|C(x) \cap C(y)| \in\left\{1, p, p^{2}\right\}$.

If $|C(x) \cap C(y)|=1$, then $C(x) \cap C(y)=\{0\}$. If $|C(x) \cap C(y)|=p$, then there exist $z \in$ $V(\Gamma(R))$ such that $z \in C(x) \cap C(y)$. By Lemmas 2.1.12 and 2.1.13. $C(x)=C(z)$ and $C(y)=C(z)$. Thus $C(x)=C(y)$, a contradiction.

If $|C(x) \cap C(y)|=p^{2}$, then $|C(x) \cap C(y)|=$ $|C(x)|$. Since $C(x) \cap C(y) \subseteq C(x), C(x) \cap$ $C(y)=C(x)$. Thus $C(x) \subseteq C(y)$. This contradicts the fact that $y \notin C(x)$.

Case 3. Let $|C(x)|=p$ and $|C(y)|=p^{2}$. Then $|C(x) \cap C(y)| \in\{1, p\}$.

If $|C(x) \cap C(y)|=1$, then $C(x) \cap C(y)=\{0\}$. If $|C(x) \cap C(y)|=p$, then $|C(x) \cap C(y)|=$ $|C(x)|$. Since $C(x) \cap C(y) \subseteq C(x), C(x) \cap$ $C(y)=C(x)$. Thus $C(x) \subseteq C(y)$. By Lemma 2.1.12. $C(y)$ is commutative. So for every $z \in C(y), z x=x z$. Thus $z \in C(x)$ and so $C(y) \subseteq C(x)$. Hence $C(y)=C(x)$, which contradicts the fact that $y \notin C(x)$. This completes the proof.

Proof of Theorem B. Let $x, y \in V(\Gamma(R))$ and $y \notin C(x)$. Then $|C(x)|,|C(y)| \in\left\{p, p^{2}\right\}$. Also by Lemma 3.1.12. $C(x) \cap C(y)=\{0\}$. Let $|C(x)|=|C(y)|=p$. If $z \in C(x), t \in C(y)$ and $z t=t z$, then by Lemma 2.1.13. $C(x)=C(z)$, $C(y)=C(t)$ and $C(z)=C(t)$. So $C(x)=C(y)$, which is impossible. Therefore $\Gamma(R)$ is the disjoint union of $\ell$ copies of the complete graph of size $p-1$. So $|V(\Gamma(R))|=\ell(p-1)$. On 
the other hand we have $|V(\Gamma(R))|=p^{3}-1$. Thus $\ell=p^{2}+p+1$. Since $\gamma\left(K_{p-1}\right)=1$, $\gamma(\Gamma(R))=p^{2}+p+1$, and $(i)$ is proved.

Suppose $|C(x)|=p$ and $|C(y)|=p^{2}$. If $z \in C(x), t \in C(y)$, then $z t \neq t z$. Therefore $\Gamma(R)$ is the disjoint union of $\ell_{1}$ copies of the complete graph of size $p-1$ and $\ell_{2}$ copies of the complete graph of size $p^{2}-1$. So $|V(\Gamma(R))|=\ell_{1}(p-1)+\ell_{2}\left(p^{2}-1\right)$. On the other hand we have $|V(\Gamma(R))|=p^{3}-1$. Thus $\ell_{1}(p-1)+\ell_{2}\left(p^{2}-1\right)=p^{3}-1$ and so $\ell_{1}+(p+1) \ell_{2}=p^{2}+p+1$. Obviously, $\gamma(\Gamma(R))=\ell_{1}+\ell_{2}$, and (ii) is proved. Finally, if $|C(x)|=|C(y)|=p^{2}$, then $|C(x)+C(y)|=$ $p^{4}$, which is impossible.

Proof of Theorem C. Let $\gamma\left(\Gamma\left(R_{i}\right)\right)=m_{i}$ and $D_{i}=\left\{v_{i 1}, v_{i 2}, \ldots, v_{i m_{i}}\right\}$ be a dominating set in $\Gamma\left(R_{i}\right)$, where $1 \leq i \leq t$. Without loss of generality, let $m_{1} \leq m_{i}$, for every $1 \leq i \leq t$. We claim that, $D=$ $\left\{\left(v_{1 j}, 0, \ldots, 0\right) \mid 1 \leq j \leq m_{1}\right\}$ is a dominating set in $\Gamma\left(\prod_{i=1}^{t} R_{i}\right)$. For let $\left(u_{1}, u_{2}, \ldots, u_{t}\right) \in$ $V\left(\Gamma\left(\prod_{i=1}^{t} R_{i}\right)\right)$ be an arbitrary vertex. Since $D_{1}$ is a dominating set in $\Gamma\left(R_{1}\right)$, there exist $v_{1 j} \in D_{1}$ such that $v_{1 j} u_{1}=u_{1} v_{1 j}$ or $v_{1 j}=u_{1}$. Thus $\left(u_{1}, u_{2}, \ldots, u_{t}\right) \in N\left(\left(v_{1 j}, 0, \ldots, 0\right)\right)$ in $\Gamma\left(\prod_{i=1}^{t} R_{i}\right)$. Let $D^{\prime}=\left\{\left(v_{1 j}, v_{2 j}, \ldots, v_{t j}\right) \mid v_{i j} \in\right.$ $\left.V\left(\Gamma\left(R_{i}\right)\right), j=1, \ldots, m_{1}-1\right\} \subseteq V\left(\Gamma\left(\prod_{i=1}^{t} R_{i}\right)\right)$ be a dominating set in $\Gamma\left(\prod_{i=1}^{t} R_{i}\right)$. Let $\left(u_{1}, u_{2}, \ldots, u_{t}\right)$ be an arbitrary vertex in $\Gamma\left(\prod_{i=1}^{t} R_{i}\right)$. Since $D^{\prime}$ is a dominating set in $\Gamma\left(\prod_{i=1}^{t} R_{i}\right)$, there exist a $v_{1 j} \in V\left(\Gamma\left(R_{1}\right)\right)$ such that $u_{1} v_{1 j}=v_{1 j} u_{1}$ or $u_{1}=v_{1 j}$. Whenever $\left\{v_{11}, v_{12}, \ldots, v_{1\left(m_{1}-1\right)}\right\}$ is a dominating set in $\Gamma\left(R_{1}\right)$. This contradicts the fact that $D_{1}$ is a dominating set in $\Gamma\left(R_{1}\right)$. Hence $D$ is a dominating set in $\Gamma\left(\prod_{i=1}^{t} R_{i}\right)$, as claimed. Therefore $\gamma\left(\Gamma\left(\prod_{i=1}^{t} R_{i}\right)\right)=\operatorname{Min}_{1 \leq i \leq t}\left(\gamma\left(\Gamma\left(R_{i}\right)\right)\right)$.

Theorem 3.1.13 Let $R_{1}$ be a non-commutative ring of order $n_{1}$ and $Z\left(R_{1}\right)=0$. Also, let $R_{2}$ be a commutative ring of order $n_{2}$. Then

$$
\gamma\left(\Gamma\left(R_{1} \times R_{2}\right)\right)=\gamma\left(\Gamma\left(R_{1}\right)\right) .
$$

Proof. Let $G=\Gamma\left(R_{1} \times R_{2}\right)$ and $G^{\prime}=\Gamma\left(R_{1}\right) \otimes$ $K_{n_{2}}$ such that the members of $V\left(K_{n_{2}}\right)$ are the elements of $R_{2}$. It is easy to see that $G \cong G^{\prime}$.
It is sufficient to prove that $\gamma\left(G^{\prime}\right)=\gamma\left(\Gamma\left(R_{1}\right)\right)$. Let $\gamma\left(\Gamma\left(R_{1}\right)\right)=m$ and $D=\left\{v_{1}, v_{2}, \ldots, v_{m}\right\}$ be a dominating set in $\Gamma\left(R_{1}\right)$. It will be claimed, $D^{\prime}=\left\{\left(v_{i}, u_{0}\right) \mid v_{i} \in D\right\}$ is a dominating set in $G^{\prime}$, where $u_{0} \in V\left(K_{n_{2}}\right)$. Let $\left(v, u^{\prime}\right) \in V\left(G^{\prime}\right)$ be an arbitrary vertex. Since $D$ is a dominating set in $\Gamma\left(R_{1}\right)$, there exist $v_{i} \in D$ such that $v_{i} v=v v_{i}$ or $v=v_{i}$. By strongly product structure, $\left(v, u^{\prime}\right) \in N\left(\left(v_{i}, u_{0}\right)\right)$. Let $D^{\prime \prime}=\left\{\left(x_{i}, u_{i}\right) \mid i=1, \ldots, m-1\right\} \subseteq V\left(G^{\prime}\right)$ be a dominating set in $G^{\prime}$. Let $(v, u)$ be an arbitrary vertex in $G^{\prime}$. Since $D^{\prime \prime}$ is a dominating set in $G^{\prime}$, there exist a $v_{i} \in V\left(\Gamma\left(R_{1}\right)\right)$ such that $(v, u)$ is adjacent to $\left(v_{i}, u_{i}\right)$. By strongly product structure, $v=v_{i}$ or $v v_{i}=v_{i} v$. whenever $\left\{v_{1}, v_{2}, \ldots, v_{m-1}\right\}$ is a dominating set in $\Gamma\left(R_{1}\right)$. This contradicts the fact that $D$ is a dominating set in $\Gamma\left(R_{1}\right)$. Thus $D^{\prime}$ is a dominating set in $G^{\prime}$, as claimed. Hence $\gamma\left(G^{\prime}\right)=\gamma\left(\Gamma\left(R_{1}\right)\right)$.

\subsection{Signed domination number in $\Gamma(R)$}

Proof of Theorem D. Let $\gamma_{s}(\Gamma(R))=n-1$. By Lemma 2.3.1. every $v \in V(\Gamma(R))$ is either isolated, an endvertex or adjacent to an endvertex. Let $v \in V(\Gamma(R))$ such that $\operatorname{deg}(v)=1$ and $v \in N(u)$. Then $C(v)=\{0, v, u\}$. Thus $u=-v$ and $\operatorname{deg}(u)=1$. Hence by Lemma 2.1.3. $\Gamma(R)$ is an empty graph or union of edges.

If $n$ is even, then $\Gamma(R)$ containing isolated vertices. Thus $\bar{\Gamma}(R)$ is a complete graph. By Theorem 2.1.10, $R \cong E$ or $R \cong F$ and $(i)$ is proved. If $n$ is odd, then $\Gamma(R)$ is a union of $\frac{n-1}{2}$ copies of $P_{2}$ and (ii) is proved. The proof of converse is simple.

Theorem 3.2.1 Let $R$ be a non-commutative ring of order $n$, where $n$ is an odd number and $|Z(R)|=$ $c \neq 0$. Then $\gamma_{s}(\Gamma(R))=n-1$ if and only if $\Gamma(R)$ is the union of $\frac{n-c}{2}$ copies of $P_{2}$.

proof. Let $\gamma_{s}(\Gamma(R))=n-1$. By Lemma 2.3.1. every $v \in V(\Gamma(R))$ is either isolated, an endvertex or adjacent to an endvertex. If $x$ is an isolated vertex in $\Gamma(R)$, then $C(x)=Z(R) \cup\{x\}$. Thus $O(x)=2$, a contradiction. Let $v \in$ $V(\Gamma(R))$ such that $\operatorname{deg}(v)=1$ and $v \in N(u)$. Then $C(v)=Z(R) \cup\{v, u\}$. Since $n$ is an odd 
number, $u=-v$ and so $\operatorname{deg}(u)=1$. Therefore $\Gamma(R)$ is the union of $\frac{n-c}{2}$ copies of $P_{2}$. The proof of converse is simple.

Theorem 3.2.2 Let the situation be as in Theorem B. Then

i) $\gamma_{s}(\Gamma(R))=2\left(p^{2}+p+1\right)$.

or

ii) $\gamma_{s}(\Gamma(R))=2\left(\ell_{1}+\ell_{2}\right)$, where $\ell_{1}$ and $\ell_{2}$ satisfy in $\ell_{1}+(p+1) \ell_{2}=p^{2}+p+1$.

proof.

i) As in the proof of Theorem $\mathrm{B}, \Gamma(R)$ is the disjoint union of $p^{2}+p+1$ copies of the complete graphs of size $p-1$. By Theorem 2.3.3. $\gamma_{s}\left(K_{p-1}\right)=2$. Hence $\gamma_{s}(\Gamma(R))=2\left(p^{2}+p+\right.$ 1 ), and (i) is proved.

ii) As in the proof of Theorem $\mathrm{B}, \Gamma(R)$ is the disjoint union of $\ell_{1}$ copies of the complete graphs of size $p-1$ and $\ell_{2}$ copies of the complete graph of size $p^{2}-1$, where $\ell_{1}+(p+1) \ell_{2}=p^{2}+p+1$. By Theorem 2.3.3. $\gamma_{s}(\Gamma(R))=2\left(\ell_{1}+\ell_{2}\right)$, and $(i i)$ is proved.

Lemma 3.2.3 Let $R$ be a non-commutative finite ring and $Z(R)=\{0\}$. If $0 \neq\left|V^{-}(\bar{\Gamma}(R))\right|=t$, then the followings are hold.

i) $\delta(\bar{\Gamma}(R)) \leq 2 t+1$

ii) $|R| \leq 4 t+2$.

proof.

i) On the contrary, let $v \in V(\bar{\Gamma}(R))$ and $\operatorname{deg}(v)=\delta(\bar{\Gamma}(R)) \geq 2 t+2$. If $N(v)=$ $\left\{v_{1}, \ldots, v_{\delta(\bar{\Gamma}(R))}\right\}$, then consider the function $f: V(G) \rightarrow\{-1,1\}$ for which $f(w)=-1$ if and only if $w \in\left\{v_{1}, v_{2}, \ldots, v_{\left\lfloor\frac{\delta(\bar{\Gamma}(R)}{2}\right\rfloor}\right\}$. Clearly, $f[w] \geq 1$, for all $w \in V(\bar{\Gamma}(R))$. So $f$ is a signed dominating function. This implies that $\left|V^{-}(\bar{\Gamma}(R))\right| \geq t+1$, contrary to assumption.

ii) On the contrary, let $|R| \geq 4 t+3$. By Lemma 2.1.1. $\delta(\bar{\Gamma}(R)) \geq 2 t+2$, which is a contradiction.

Theorem 3.2.4 Let $R$ be a non-commutative ring of order $n$ and $Z(R)=\{0\}$. Then $\gamma_{s}(\bar{\Gamma}(R)) \notin$ $\{n-1, n-5\}$.

proof. On the contrary, let $\gamma_{s}(\bar{\Gamma}(R)) \in\{n-$ $1, n-5\}$. We consider the following two cases.
Case 1. Let $\gamma_{s}(\bar{\Gamma}(R))=n-1$. If $n=4$, then $\bar{\Gamma}(R)=K_{3}$ and so $\gamma_{s}(\bar{\Gamma}(R)) \neq n-1$. By corollary 2.1.7. $n \geq 8$. Since $\bar{\Gamma}(R)$ is a connected graph, by Lemma 2.3.1. every $v \in \bar{\Gamma}(R)$ is an endvertex or adjacent to an endvertex. We claim that $\bar{\Gamma}(R)$ have exactly one vertex with degree greater than 1 .

Suppose that $\operatorname{deg}(u), \operatorname{deg}(v)>1$. So there are $x, y \in V(\bar{\Gamma}(R))$ such that $x \in N(u)$ and $y \in N(v)$. Since $\bar{\Gamma}(R)$ is a connected graph and by theorem 2.1.4 $\operatorname{diam}(\bar{\Gamma}(R))=2, v=u$ and $x-v-u$. Hence $\bar{\Gamma}(R) \cong K_{1, n}$, contrary to Theorem 2.1.5. Therefore $\gamma_{s}(\bar{\Gamma}(R)) \neq n-1$.

Case 2. Let $\gamma_{s}(\bar{\Gamma}(R))=n-5$. Then $\left|V^{-}(\bar{\Gamma}(R))\right|=2$. By Lemma 3.2.3. $\delta(\bar{\Gamma}(R)) \leq 5$ and $n \leq 10$. By Corrolary 2.1.7 $n \geq 8$. By Lemmas 2.3.5 and 2.3.6. $n \notin\{8,10\}$. Let $n=9$ and $v \in V(\bar{\Gamma}(R))$ such that $\operatorname{deg}(v)=k=\delta(\bar{\Gamma}(R))$. By Lemma 2.1.1 $k \geq 5$ and so $\delta(\bar{\Gamma}(R))=5$. Hence $|C(v)| \nmid 9$, a contradiction. Therefore $\gamma_{s}(\bar{\Gamma}(R)) \neq n-5$.

Theorem 3.2.5 Let $R$ be a non-commutative ring of order $n$ and $Z(R)=\{0\}$. Then $\gamma_{s}(\bar{\Gamma}(R))=$ $n-3$ if and only if $R$ is isomorphic with one of the following rings

$E=\langle x, y| 2 x=2 y=0, x^{2}=x, y^{2}=y, x y=$ $x, y x=y\rangle$

$F=\langle x, y| 2 x=2 y=0, x^{2}=x, y^{2}=y, x y=$ $y, y x=x\rangle$.

Proof. Let $\gamma_{s}(\bar{\Gamma}(R))=n-3$. Then $\left|V^{-}(\bar{\Gamma}(R))\right|=1$. By Corrolary 2.1.7 and Lemma 3.2.3. $n=4$ and $\delta(\bar{\Gamma}(R))=2$. Thus $\bar{\Gamma}(R)=K_{3}$. Hence by Theorem 2.1.10, $R$ is one of the following rings

$E=\langle x, y| 2 x=2 y=0, x^{2}=x, y^{2}=y, x y=$ $x, y x=y\rangle$

$F=\langle x, y| 2 x=2 y=0, x^{2}=x, y^{2}=y, x y=$ $y, y x=x\rangle$.

The proof of converse is straightforward.

Corollary 3.2.6 Let the situation be as in 3.2.5 If $\gamma_{s}(\bar{\Gamma}(R))=n-3$, then $\gamma(\bar{\Gamma}(R))=1$.

\section{Proof of Theorem E.}

Let $v_{i} \in V\left(\Gamma\left(R_{i}\right)\right)$ and $\operatorname{deg}\left(v_{i}\right)=\delta_{i}$, where $1 \leq i \leq t$. Since $Z\left(R_{i}\right)=\{0\}, Z\left(\prod_{i=1}^{t} R_{i}\right)=$ $0 . \quad$ If $\left(v_{1}, v_{2}, \ldots, v_{t}\right) \in V\left(\Gamma\left(\prod_{i=1}^{t} R_{i}\right)\right)$, then $N\left[\left(v_{1}, v_{2}, \ldots, v_{t}\right)\right]=\left\{\left(x_{1}, x_{2}, \ldots, x_{t}\right) \mid\right.$ 
$\left.x_{i} \in N\left[v_{i}\right] \cup\{0\}\right\} \backslash\{(0,0, \ldots, 0)\} . \quad$ Thus $\operatorname{deg}\left(\left(v_{1}, v_{2}, \ldots, v_{t}\right)\right)=\left(\prod_{i=1}^{t}\left(\delta_{i}+2\right)\right)-2$. Also $\left(v_{1}, v_{2}, \ldots, v_{t}\right)$ is a vertex of minimum degree in $\Gamma\left(\prod_{i=1}^{t} R_{i}\right)$. Let $\delta=\left(\prod_{i=1}^{t}\left(\delta_{i}+2\right)\right)-2$ and use $u_{i} \in V\left(\Gamma\left(\prod_{i=1}^{t} R_{i}\right)\right), 1 \leq i \leq \delta$, to denote the neighbors of $\left(v_{1}, v_{2}, \ldots, v_{t}\right)$. Consider the function $f: V\left(\Gamma\left(\prod_{i=1}^{t} R_{i}\right)\right) \rightarrow\{-1,1\}$ for which $f\left(u_{j}\right)=-1$ and $f\left(u_{k}\right)=1$, such that $1 \leq j \leq\left\lfloor\frac{\delta}{2}\right\rfloor$ and $\left\lfloor\frac{\delta}{2}\right\rfloor+1 \leq$ $k \leq \delta$. Also, for each $u \in V\left(\Gamma\left(\prod_{i=1}^{t} R_{i}\right)\right)$ such that $u \neq u_{i}, f(u)=1$. Clearly, $f[w] \geq 1$, for all $w \in V\left(\Gamma\left(\prod_{i=1}^{t} R_{i}\right)\right)$. Therefore $\mathrm{f}$ is a signed dominating function. Hence $\left|V^{-}\left(\Gamma\left(\prod_{i=1}^{t} R_{i}\right)\right)\right| \geq\left\lfloor\frac{\delta}{2}\right\rfloor$. Since $\gamma_{s}\left(\Gamma\left(\prod_{i=1}^{t} R_{i}\right)\right)=\left|V\left(\Gamma\left(\prod_{i=1}^{t} R_{i}\right)\right)\right|-$ $2\left|V^{-}\left(\Gamma\left(\prod_{i=1}^{t} R_{i}\right)\right)\right|$, it follows that $\gamma_{s}\left(\Gamma\left(\prod_{i=1}^{t} R_{i}\right)\right) \leq\left(\prod_{i=1}^{t} n_{i}\right)-1-2\left\lfloor\frac{\delta}{2}\right\rfloor$. We consider the following two cases.

Case 1. Let $\delta$ be odd. Then $\gamma_{s}\left(\Gamma\left(\prod_{i=1}^{t} R_{i}\right)\right) \leq$ $\left(\prod_{i=1}^{t} n_{i}\right)-1-2\left\lfloor\frac{\delta}{2}\right\rfloor . \quad$ It follows that $\gamma_{s}\left(\Gamma\left(\prod_{i=1}^{t} R_{i}\right)\right) \leq\left(\prod_{i=1}^{t} n_{i}\right)-1-2\left\lfloor\frac{\delta-1}{2}\right\rfloor$. Hence $\gamma_{s}\left(\Gamma\left(\prod_{i=1}^{t} R_{i}\right)\right) \leq\left(\prod_{i=1}^{t} n_{i}\right)-\delta$ and (i) is proved.

Case 2. Let there exist $1 \leq i \leq t$ such that $\delta_{i}$ be even. Then $\gamma_{s}\left(\Gamma\left(\prod_{i=1}^{t} R_{i}\right)\right) \leq\left(\prod_{i=1}^{t} n_{i}\right)-1-\delta$ and (ii) is proved.

\section{REFERENCES}

[1] S. Akbari, H. Bidkhori and A. Mohammadian, (2008), Commuting graph of Matrix Algebra, Communication in Algebra, 36: 4020-4031.

[2] S. Akbari, M. Ghandehari, H. Radjavi, M. Hadadian and A. Mohammadian, (2004), On the commuting graphs of semisimple rings, Linear Algebra Appl, 345-355.

[3] S. Akbari, A. Mohammadian, H. Radjavi, P. Raja, (2006), On the diameters of commuting graphs, Linear Algebra and its Application, 161-176.

[4] S. Akbari, P. Raja, (2006), Commuting graphs of some subsets in simple rings, Linear Algebra APPL., 416:1038-1047.

[5] T. J. Bean, M. A. Henning, and H. C. Swart, (1994), On the integrity of distance dom- ination in graphs, Australas. J. Combin., 10: $29-43$.

[6] W. Chen, E. Song, (2006), Lower bounds on several version of signed domination number, Discrete Mathematics, 1837-1846.

[7] J. F. Fink, M. S. Jacobson, L. F. Kinch and J. Roberts, (1985), On graphs having domination number half their order, Period. Math. Hunger., 16: 287-293.

[8] P. Flach, L. Volkmann, (1990), Estimations for the domination number of a graph, Discrete Math, 145-151.

[9] S. Gravier, A. Khelladi, (1995), On the domination number of cross products of graphs, Discrete Mathematics, 145: 273277.

[10] Ruth Haas, Thomas B. Wexler, (2002), Bounds on the signed domination number of a graph, Electronic Notes in Discrete Mathematics, 742-750.

[11] Ruth Haas, Thomas B. Wexler, (2004), Signed domination number of a graph and its complement, Discrete Mathematics, 87-92.

[12] W. Haynes, T. Hedetniemi, J. Slater, (1998), Fundementals of domination in graphs, MARCEL DEKKER INC..

[13] F. Jaeger, C. Payan, (1972), Relation du type Nordhaus-Gaddum pour le numbered absorption dun graphe simple, C. R. Acard. Sci. Paris, 274: 728-730.

[14] G. R. Omidi, E. Vatandoost, (2011), On the commuting graph of rings, Journal of Algebra and It's Applications, 10: 521-527.

[15] O. Ore, (1962), Theory of Graphs, Amer. Math. Soc. Colloq. PubL, 38 (Amer. Math. Soc, Providence, RI).

[16] C. Payan, (1975), Sur le nombre d'absorption d'un graphe simple, Cahiers Centre Etudes Rech. Oper. B.3.4, 171. 
[17] G. Scorza, (1926), I gruppi che possono pensarsi come somma di tre loro sottogruppi, Boll. Un. Mat. Ital., 5: 216-218.

[18] H. B. Walikar, B. D. Acharya, and E. Sam- pathkumar, (1979), Recent develop- developments in the theory of domination in graphs, In MRI Lecture Notes in Math., Mahta Research Instil, Allahabad, volume 1. 